\title{
The Practical Aspects in the Determination of Membrane Properties for Gas Permeation
}

\author{
Haoyu Wu, Boguslaw Kruczek and Jules Thibault \\ University of Ottawa, Department of Chemical and Biological Engineering \\ 161 Louis Pasteur, Ottawa, Ontario, Canada, K1N 6N5 \\ hwu055@uottawa.ca; bkruczek@uottawa.ca; Jules.Thibault@uottawa.ca
}

\section{Extended Abstract}

Membrane-based pressure driven processes have been widely studied and have been used in a myriad of applications. The objective of the characterization of membranes in which gas sorption obeys Henry's law is to determine the solubility $S$ and diffusivity $D$ of the membrane. The time-lag method, developed by Daynes [1] and Barrer [2], is commonly used as the integral approach [3] in the characterization of membranes by measuring the cumulated amount of permeated gas after a sudden change of the upstream boundary condition [3]. One of the important assumptions of the time-lag method is that the amount of permeate gas accumulating in the downstream receiver is small enough to have a negligible effect on the driving force. To better satisfy this assumption, it was claimed that a capacity parameter (related to the receiver volume) should be small enough [4]. Nevertheless, due to the existence of measurement noise in experimental data, the accurate determination of the time lag is difficult and a small capacity parameter will intensify the effect of noise. The accuracy of the estimated time lag is also influenced by the noise level and the data analysis procedure. An alternative to obtain the membrane properties is to fit the variation of the pressure change in the downstream reservoir as a function of time using the nonlinear regression method [5]. By minimizing the sum of squares of the differences between the experimental data and the predictions from the numerical model, the best combinations of the membrane properties can be obtained. The latter method also allows using the real rather than ideal boundary conditions at the membrane interfaces.

In this paper, the experimental results are obtained from the constant volume membrane system which consists of two fixed volumes separated by a membrane cell module [7]. The system is initially evacuated using a vacuum pump prior to each experiment. The permeation process is initiated by performing a step change of the gas pressure at the upstream side of the membrane. The progressive permeation of the gas within the membrane leads to a pressure increase at the downstream side of the membrane which is recorded by a high precision pressure transducer. The time lag can be determined from the time-axis intercept of the quasi steady-state portion of the pressure rise curve plotted versus time. To gain a better understanding of the gas permeation process, a numerical model was also used to simulate the real experimental process and to predict the behaviours of membranes and various boundary conditions $[9,10]$.

In this paper, practical suggestions for the determination of membrane properties are given based on the commonly used time-lag method and on the nonlinear regression method. For the time-lag method, the major sources of noise were analysed and the level of noise as well as the length of the evaluation window were evaluated to estimate the accuracy of time lag results. Suggestions are given based on how to reduce the noise by properly selecting the design parameters of the experiments, such as the downstream volume, and the data analysis. For the nonlinear regression method, results show that it is nearly impossible to recover the real values of the membrane permeation properties, i.e. $S, D$ due to the very strong correlations that prevail between $S$ and $D$. Instead of exploring the effect of individual values of the membrane properties, combinations of the property coefficients that lead to same accuracy were studied. Contour maps and suggestions on the relative weights of each section of the pressure rise versus time curve are provided in the paper to assist researchers to better use the characterization method of the membrane.

Keywords: Gas membrane permeation, noise, time lag, nonlinear least square, diffusivity. 


\section{References}

[1] R. M. Barrer and E. K. Rideal, "Permeation, diffusion and solution of gases in organic polymers," Trans. Faraday Soc. Transactions of the Faraday Society, vol. 35, pp. 628-643, 1939.

[2] H. A. Daynes, "The Process of Diffusion through a Rubber Membrane," in Proceedings of the Royal Society A: Mathematical, Physical and Engineering Sciences (1905-1934), vol. 97, no. 685, pp. 286-307, 1920.

[3] R. M. Felder, "Estimation of gas transport coefficients from differential permeation, integral permeation, and sorption rate data," J. Memb. Sci., vol. 3, no. 1, pp. 15-27, 1978.

[4] P. Taveira, A. Mendes, and C. Costa, "On the determination of diffusivity and sorption coefficients using different timelag models," J. Memb. Sci., vol. 221, no. 1-2, pp. 123-133, 2003.

[5] D. M. Himmelblau, Process analysis by statistical methods. New York: Wiley, 1970.

[6] N. Al-Qasas, J. Thibault, and B. Kruczek, "The effect of the downstream pressure accumulation on the time-lag accuracy for membranes with non-linear isotherms," J. Membr. Sci. under review.

[7] M. Al-Ismaily, J.G. Wijmans, and B. Kruczek, "A shortcut method for faster determination of permeability coefficient from time lag experiments," J. Membr. Sci., vol. 423-424, pp. 165-174, 2012.

[8] S. W. Rutherford and D. D. Do, "Review of time lag permeation technique as a method for characterisation of porous media and membranes," Adsorption, vol. 3, pp. 283-312, 1997

[9] H. Wu, N. Al-Qasas, B. Kruczek, and J. Thibault, "Simulation of Time-Lag Permeation Experiments Using Finite Differences," J. Fluid Flow, Heat Mass Transf., vol. 2, no. 181, pp. 1-17, 2015.

[10] B. Carnahan, H.A. Luther, and J. O. Wilkes, Applied numerical methods. New York, USA: Wiley, 1969. 